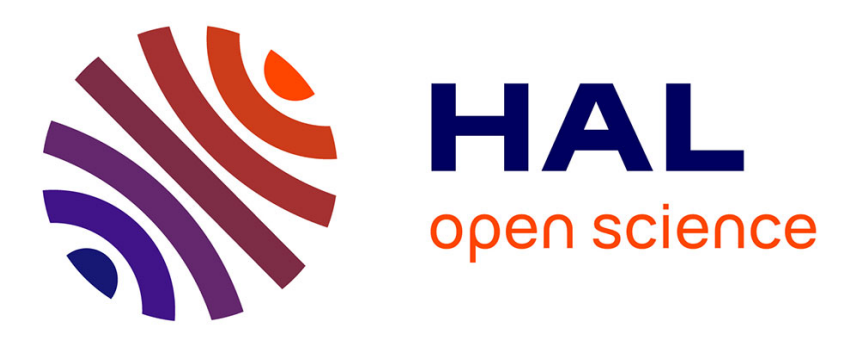

\title{
A modified phase-fitted and amplification-fitted Runge-Kutta-Nyström method for the numerical solution of the radial Schrödinger equation
}

D. F. Papadopoulos, Z. A. Anastassi, T. E. Simos

\section{- To cite this version:}

D. F. Papadopoulos, Z. A. Anastassi, T. E. Simos. A modified phase-fitted and amplification-fitted Runge-Kutta-Nyström method for the numerical solution of the radial Schrödinger equation. Journal of Molecular Modeling, 2010, 16 (8), pp.1339-1346. 10.1007/s00894-009-0626-7 . hal-00562268

\section{HAL Id: hal-00562268 \\ https://hal.science/hal-00562268}

Submitted on 3 Feb 2011

HAL is a multi-disciplinary open access archive for the deposit and dissemination of scientific research documents, whether they are published or not. The documents may come from teaching and research institutions in France or abroad, or from public or private research centers.
L'archive ouverte pluridisciplinaire HAL, est destinée au dépôt et à la diffusion de documents scientifiques de niveau recherche, publiés ou non, émanant des établissements d'enseignement et de recherche français ou étrangers, des laboratoires publics ou privés. 


\section{Editorial Manager(tm) for Journal of Molecular Modeling Manuscript Draft}

\section{Manuscript Number: JMM01019R1}

Title: A modified phase-fitted and amplification-fitted Runge-Kutta-Nystr $\backslash$ " $\{0\}$ m method for the numerical solution of the Radial Schr $\backslash$ "\{o\}dinger equation

Article Type: Original paper

Keywords: Runge-Kutta-Nystr $\backslash$ "\{o\}m methods; Phase-fitted; Amplification-fitted; Schr $\backslash$ "\{o\}dinger; Phase-lag infinity

Corresponding Author: Prof. Theodore Simos,

Corresponding Author's Institution:

First Author: D. F Papadopoulos

Order of Authors: D. F Papadopoulos; Z. A Anastassi; Theodore Simos

Abstract: A new Runge-Kutta-Nystr $\backslash$ " $\{0\}$ m method, with phase-lag and amplification error of order infinity, for the numerical solution of the Schr $\backslash$ "\{o\}dinger equation is developed in this paper. The new method is based on the Runge-Kutta-Nystr $\backslash\{0\}$ m method with fourth algebraic order, developed by Dormand, El-Mikkawy and Prince. Numerical illustrations indicate that the new method is much more efficient than other methods derived for the same purpose.

Response to Reviewers: Reviewer \#1: The authors modified the fourth order Runge-Kutta-Nystrom method. The numerical approach to me is OK. However, I think that efficiency evaluation of the method is too brief and more measurement is needed for a complete comparison with other methods. I suggest the authors use other ways to evaluate the performance of the new method. Moreover, it is of interest to know the efficiency at different orders.

We have read the reviewer's comments and we made the following changes:

- We added the bound states problem using eight eigenenergies. We present the figures with the efficiency of the methods for these eigenenergies and some remarks on the results. There are no other RKN methods of the same order that are used for the solution of the Schrodinger equation so that we can compare with.

We have also changed Theorem 1, so that it is more clear and precise. 


\section{A modified phase-fitted and amplification-fitted Runge- Kutta-Nyström method for the numerical solution of the Radial Schrödinger equation}

D. F. Papadopoulos*

Department of Computer Science and Technology, Faculty of Sciences and Technology, University of Peloponnese, GR-22 100 Tripolis, Greece

\section{Z. A. Anastassi ${ }^{\dagger}$}

Department of Finance and Auditing, School of Management and Economics, Technological Educational Institute of Kalamata, GR-241 00 Antikalamos, Greece

\section{T. E. Simos ${ }^{\ddagger}$}

KFUPM Chair Professor, Department of Mathematics and Statistics, King Fahd University of Petroleum and Minerals, P. O. Box 5046, Dhahran 31261, Saudi Arabia and Laboratory of Computational Science, Department of Computer Science and Technology, Faculty of Sciences and Technology, University of Peloponnese, GR-22 100 Tripolis, Greece

Abstract. A new Runge-Kutta-Nyström method, with phase-lag and amplification error of order infinity, for the numerical solution of the Schrödinger equation is developed in this paper. The new method is based on the Runge-Kutta-Nyström method with fourth algebraic order, developed by Dormand, El-Mikkawy and Prince. Numerical illustrations indicate that the new method is much more efficient than other methods derived for the same purpose.

Keywords: Runge-Kutta-Nyström methods, Phase-fitted, Amplification-fitted, Schrödinger, Phase-lag infinity.

PACS: $0.260,95.10 . \mathrm{E}$

\section{Introduction}

The one-dimensional or radial Schrödinger equation has the form

$$
y^{\prime \prime}(x)+\left(E-\frac{l(l+1)}{x^{2}}-V(x)\right) y(x)=0, \quad \text { where } \quad 0 \leq x<\infty
$$

\footnotetext{
* e-mail: dimfpap@gmail.com

$\dagger$ e-mail: zackanas@gmail.com

$\ddagger$ Highly Cited Researcher, Active Member of the European Academy of Sciences and Arts, Corresponding Member of the European Academy of Sciences, Corresponding Member of European Academy of Arts, Sciences and Humanities, Address: Dr. T.E. Simos, 10 Konitsis Street, Amfithea - Paleon Faliron, GR-175 64 Athens, GREECE, Tel: 00302109421 510, e-mail: tsimos.conf@gmail.com
} 
We call the term $l(l+1) / x^{2}$ the centrifugal potential, and the function $V(x)$ the electric potential. In (1), $E$ is a real number denoting the energy, and $l$ is a quantum number. The function $W(x)=l(l+1) / x^{2}+$ $V(x)$ denotes the effective potential, where $\lim _{x \rightarrow \infty} V(x)=0$ and so $\lim _{x \rightarrow \infty} W(x)=0$. The boundary condition are $y(0)=0$ together with a second boundary condition, for large values of $\mathrm{x}$, determined by the physical considerations.

This type of equations appears in many scientific areas including, theoretical physics, nuclear physics, molecular physics, physical chemistry, quantum chemistry. For the numerical solution of equation (1) there is a high demand. The last three decades a lot of researchers have developed new methods to solve numerically the Schrödinger equation $[3-14]$.

The purpose of this paper is to construct an explicit Runge-KuttaNyström with fourth algebraic order, phase lag of order infinity and amplification error of order infinity. The new method is based on the coefficients of the well-known fourth algebraic order Dormand, El-Mikkawy and Prince Runge-Kutta-Nyström method [1]. It is also based on the FSAL (first stage as last) technique, which means that the number of stages is four but it effectively uses only three stages at each step.

\section{Modified Runge-Kutta-Nyström method}

The general m-stage method for the equation

$$
\frac{d^{2} u(t)}{d t^{2}}=f(t, u(t))
$$

is of the form

$$
\begin{array}{r}
u_{n}=u_{n-1}+h \hat{u}_{n-1}+h^{2} \sum_{i=1}^{m} b_{i} f\left(t_{n-1}+c_{i} h, f_{i}\right), \\
\hat{u}_{n}=\hat{u}_{n-1}+h \sum_{i=1}^{m} \hat{b}_{i} f\left(t_{n-1}+c_{i} h, f_{i}\right),
\end{array}
$$

where

$$
f_{i}=u_{n-1}+h g_{i} c_{i} \hat{u}_{n-1}+h^{2} \sum_{j=1}^{i-1} \alpha_{i j} f\left(t_{n-1}+c_{j} h, f_{j}\right), \quad i=1, \ldots, m
$$

especially for the explicit Runge-Kutta-Nyström methods $c_{1}=0$ and more specifically for an FSAL explicit RKN method $c_{m}=1$ and $c_{m, j}=$ $b_{i}$ for $j=i$. 
Table I. m-stage Runge-Kutta-Nystöm method

\begin{tabular}{c|c|ccccc}
$c_{1}$ & 1 & 0 & & & & \\
$c_{2}$ & $g_{2}$ & $\alpha_{21}$ & & & & \\
$c_{3}$ & $g_{3}$ & $\alpha_{31}$ & $\alpha_{32}$ & & & \\
$\vdots$ & $\vdots$ & $\vdots$ & $\vdots$ & & & \\
$c_{m}$ & $g_{m}$ & $\alpha_{m, 1}$ & $\alpha_{m, 2}$ & $\ldots$ & $\alpha_{m, m-1}$ & \\
\hline & & $b_{1}$ & $b_{2}$ & $\ldots$ & $b_{m-1}$ & $b_{m}$ \\
& & $\hat{b}_{1}$ & $\hat{b}_{2}$ & $\ldots$ & $\hat{b}_{m-1}$ & $\hat{b}_{m}$
\end{tabular}

The above expressions are presented in Table I.

As we can see in Table I, the Butcher table has an extra column as compared to a classical m-stage Runge-Kutta-Nyström method. For the classical method $g_{i}=1, i=2(1) \mathrm{m}$. In the present paper and based on the requirement of phase and amplification fitting, values $g_{i}, i=2(1) m$ are variable and depend on $z$ (which is the product of the frequency $v$ and the step-size $h$ ). In section (4) we will present a development of a phase and amplification fitted four-stage Runge-Kutta-Nyström method of algebraic order four, based on the well-known DEP RungeKutta-Nyström [1].

\section{Phase lag and amplification error analysis for Runge-Kutta-Nyström methods}

To develop the new method we use the test equation,

$$
\frac{d^{2} u(t)}{d t^{2}}=(i v)^{2} u(t) \Longrightarrow u^{\prime \prime}(t)=-v^{2} u(t), \quad v \in R
$$

By applying the general method (3) to the test equation (5) we obtain the numerical solution

$$
\left[\begin{array}{c}
u_{n} \\
h \hat{u}_{n}
\end{array}\right]=D^{n}\left[\begin{array}{c}
u_{0} \\
h \hat{u}_{0}
\end{array}\right], \quad D=\left[\begin{array}{cc}
A\left(z^{2}\right) & B\left(z^{2}\right) \\
\dot{A}\left(z^{2}\right) & \dot{B}\left(z^{2}\right)
\end{array}\right], \quad z=v h
$$

where $A, B, \dot{A}, \dot{B}$ are polynomials in $z^{2}$, completely determined by the parameters of the method (3).

The exact solution of (5) is given by

$$
u\left(t_{n}\right)=\sigma_{1}[\exp (i z)]^{n}+\sigma_{2}[\exp (-i z)]^{n},
$$

where

$$
\sigma_{1,2}=\frac{1}{2}\left[u_{0} \pm \frac{\left(i \hat{u}_{0}\right)}{v}\right] \quad \text { or } \quad \sigma_{1,2}=|\sigma| \exp ( \pm i \chi)
$$


Substituting in (7), we have

$$
u\left(t_{n}\right)=2|\sigma| \cos (\chi+n z) .
$$

Furthermore we assume that the eigenvalues of $D$ are $\varrho_{1}, \varrho_{2}$, and the consequent eigenvectors are $\left[1, v_{1}\right]^{T},\left[1, v_{2}\right]^{T}$,

where $v_{i}=\dot{A} /\left(\rho_{i}-\dot{B}\right), i=1,2$. The numerical solution of (5) is

$$
u_{n}=c_{1} \rho_{1}^{n}+c_{2} \rho_{2}^{n}
$$

where

$$
c_{1}=-\frac{v_{2} u_{0}-h \hat{u}_{0}}{v_{1}-v_{2}}, \quad c_{2}=\frac{v_{1} u_{0}-h \hat{u}_{0}}{v_{1}-v_{2}} .
$$

If $\rho_{1}, \rho_{2}$ are complex conjugate, then $c_{1,2}=|c| \exp ( \pm i w)$ and $\rho_{1,2}=$ $|\rho| \exp ( \pm i p)$. By substituting in (9), we have

$$
u_{n}=2|c||\rho|^{n} \cos (w+n p) .
$$

The following definition is originally formulated by van der Houwen and Sommeijer [2].

DEFINITION 1. (Phase-lag). Apply the RKN method (3) to the general method (5). Then we define the phase-lag

$$
\Phi(z)=z-\arccos (\operatorname{tr}(D) / 2 \sqrt{\operatorname{det}(D)})
$$

. If $\Phi(z)=O\left(z^{q+1}\right)$, then the RKN method is said to have phaselag order $q$. In addition, the quantity $a(z)=1-\sqrt{\operatorname{det}(D)}$ is called amplification error.

Let us denote

$$
\begin{aligned}
& R\left(z^{2}\right)=\operatorname{tr}(D)=A\left(z^{2}\right)+\dot{B}\left(z^{2}\right) \\
& Q\left(z^{2}\right)=\operatorname{det}(D)=A\left(z^{2}\right) \dot{B}\left(z^{2}\right)-\dot{A}\left(z^{2}\right) B\left(z^{2}\right)
\end{aligned}
$$

where $z=v h$. From definition 1 it follows that

$$
\Phi(z)=z-\arccos \left(\frac{R\left(z^{2}\right)}{2 \sqrt{Q\left(z^{2}\right)}}\right), \quad a(z)=1-\sqrt{Q\left(z^{2}\right)} .
$$

If at a point $z, a(z)=0$, then the Runge-Kutta-Nyström method has zero dissipation at this point.

We can also put forward an alternative definition for the case of infinite order of phase lag. 
DEFINITION 2. (Phase-lag of order infinity). To obtain phase-lag of order infinity the relation $\Phi(z)=z-\arccos \left(\frac{R\left(z^{2}\right)}{2 \sqrt{Q\left(z^{2}\right)}}\right)=0$ must be hold.

From definition 2 we have the following theorem.

THEOREM 1. If we have phase-lag of order infinity and at a point $z$, $\alpha(z)=0$ then,

$$
\left.\begin{array}{l}
z-\arccos \left(\frac{R\left(z^{2}\right)}{2 \sqrt{Q\left(z^{2}\right)}}\right)=0 \\
1-\sqrt{Q\left(z^{2}\right)}=0
\end{array}\right\} \Rightarrow \begin{aligned}
& R\left(z^{2}\right)=2 \cos (z) \\
& Q\left(z^{2}\right)=1
\end{aligned}
$$

Proof.

$$
\begin{array}{r}
\Phi(z)=z-\arccos \left(\frac{R\left(z^{2}\right)}{2 \sqrt{Q\left(z^{2}\right)}}\right)=0 \Leftrightarrow \\
\arccos \left(\frac{R\left(z^{2}\right)}{2 \sqrt{Q\left(z^{2}\right)}}\right)=z \Leftrightarrow \\
\cos \left(\arccos \left(\frac{R\left(z^{2}\right)}{2 \sqrt{Q\left(z^{2}\right)}}\right)\right)=\cos (z) \Leftrightarrow \\
\left(\frac{R\left(z^{2}\right)}{2 \sqrt{Q\left(z^{2}\right)}}\right)=\cos (z) \Leftrightarrow \\
\left(\frac{R\left(z^{2}\right)}{\sqrt{Q\left(z^{2}\right)}}\right)=2 \cos (z) .
\end{array}
$$

Now for

$$
a(z)=0 \Leftrightarrow 1-\sqrt{Q\left(z^{2}\right)}=0 \Leftrightarrow Q\left(z^{2}\right)=1 .
$$

From the above relation and the expression (13) we have

$$
R\left(z^{2}\right)=2 \cos (z) .
$$

LEMMA 1. For the construction of a method with phase lag of order infinity and amplification error of order infinity, we must sutisfy the conditions $R\left(z^{2}\right)=2 \cos (z)$ and $Q\left(z^{2}\right)=1$. 
Table II. Fourth-order explicit Runge-Kutta-Nystöm method

\begin{tabular}{c|c|cccc}
0 & 1 & 0 & & & \\
$\frac{1}{4}$ & $g_{2}$ & $\frac{1}{32}$ & & & \\
$\frac{7}{10}$ & $g_{3}$ & $\frac{7}{1000}$ & $\frac{119}{500}$ & & \\
1 & 1 & $\frac{1}{14}$ & $\frac{8}{27}$ & $\frac{25}{189}$ & \\
\hline & & $\frac{1}{14}$ & $\frac{8}{27}$ & $\frac{25}{189}$ & 0 \\
& & $\frac{1}{14}$ & $\frac{32}{81}$ & $\frac{250}{567}$ & $\frac{5}{54}$
\end{tabular}

\section{Derivation of the new Runge-Kutta-Nyström method}

In this section we construct a four-stage explicit Runge-Kutta-Nyström method (presented in table I), based on $R\left(z^{2}\right)=2 \cos (z)$ and $Q\left(z^{2}\right)=1$. Using the coefficients of DEP [1] and for $g_{4}=1$, the method can be seen in table II.

Now let us rewrite $R\left(z^{2}\right)$ and $Q\left(z^{2}\right)$ in the following form

$$
\begin{aligned}
& R\left(z^{2}\right)=2-r_{1} z^{2}+r_{2} z^{4}-r_{3} z^{6}+\ldots+r_{i} z^{2 i}=0 \\
& Q\left(z^{2}\right)=1-q_{1} z^{2}+q_{2} z^{4}-q_{3} z^{6}+\ldots+q_{i} z^{2 i}=0
\end{aligned}
$$

By computing the polynomials $A, \dot{A}, B, \dot{B}$ in terms of RKN parameters we obtain the expressions of $R\left(z^{2}\right)$ and $Q\left(z^{2}\right)$. Then by applying them to the equations (14) and (15) we lead to the following formulas for the coefficients $g_{2}$ and $g_{3}$.

$$
\begin{aligned}
g_{2} & =-1 / 20\left(-933120+459 z^{6}-19440 z^{4}+276480 z^{2}-18(-597196800\right. \\
& +597196800 \cos (z)-576020 z^{8}+12913920 z^{6}-98858880 z^{4} \\
& \left.+349401600 z^{2}+8551 z^{10}+7102080 z^{4} \cos (z)-50803200 z^{2} \cos (z)\right) \\
& \left./\left(-186624+31104 z^{2}-1332 z^{4}+17 z^{6}\right)\right)+648((-597196800 \\
& +597196800 \cos (z)-576020 z^{8}+12913920 z^{6}-98858880 z^{4} \\
& \left.+349401600 z^{2}+8551 z^{10}+7102080 z^{4} \cos (z)-50803200 z^{2} \cos (z)\right) \\
& \left./\left(z^{2}\left(-186624+31104 z^{2}-1332 z^{4}+17 z^{6}\right)\right)\right) \\
& +933120 \cos (z) z^{-2}\left(17 z^{4}-772 z^{2}+2304\right)^{-1} \\
& \\
g_{3} & =\frac{9}{2000}\left(-597196800+597196800 \cos (z)-576020 z^{8}+12913920 z^{6}\right. \\
& -98858880 z^{4}+349401600 z^{2}+8551 z^{10}+7102080 z^{4} \cos (z)
\end{aligned}
$$


$\left.-50803200 z^{2} \cos (z)\right) /\left(z^{4}\left(-186624+31104 z^{2}-1332 z^{4}+17 z^{6}\right)\right)$

When $z<0.5$ the following Taylor series expansions are used

$g_{2}=1-\frac{43}{1152} z^{2}+\frac{13}{17920} z^{4}-\frac{2603}{209018880} z^{6}-\frac{22681}{14780620800} z^{8}-\frac{1039043569}{5423187138969600} z^{10}$
$g_{3}=1+\frac{43}{3600} z^{2}-\frac{2183}{4838400} z^{4}-\frac{1249}{373248000} z^{6}-\frac{3577129}{4138573824000} z^{8}-\frac{943612843}{8473729904640000} z^{10}$

\section{Numerical illustrations}

In this section we will apply our method to the radial Schrödinger equation. For the purpose of our numerical illustration we take the domain of integration as $x \in[0,15]$, using the Woods-Saxon potential:

$$
\begin{array}{r}
V(x)=\frac{u_{0}}{1+q}+\frac{u_{1} q}{(1+q)^{2}}, \quad q=\exp \left(\frac{x-x_{0}}{\alpha}\right), \quad \text { where } \\
u_{0}=-50, \quad \alpha=0.6, \quad x_{0}=7, \quad u_{1}=-\frac{u_{0}}{\alpha}
\end{array}
$$

\subsection{Resonanse PRoblem}

In the case of positive energies $\left(E=k^{2}\right)$, the potential $(V(x))$ dies away faster than the centrifugal potential $\left(l(l+1) / x^{2}\right)$, so for a large number for $x$, Schrödinger equation effectively reduces to

$$
y^{\prime \prime}(x)+\left(k^{2}-\frac{l(l+1)}{x^{2}}\right) y(x)=0
$$

The above equation (18) has two linearly independent solutions, $k x j_{l}(k x)$ and $k x n_{l}(k x)$, where $j_{l}$ and $n_{l}$ are the spherical Bessel and Neumann functions, respectively. When $x \rightarrow \infty$, the solution of equation (1) take the following asymptotic form

$$
\begin{array}{r}
y(x) \simeq A k x j_{l}(k x)-B k x n_{l}(k x) \\
\simeq D\left[\sin \left(k x-\frac{l \pi}{2}\right)+\tan \left(\delta_{l}\right) \cos \left(k x-\frac{l \pi}{2}\right)\right],
\end{array}
$$

where $\delta_{l}$ is the scattering phase shift that may be calculated from the bellow formula 


$$
\tan \left(\delta_{l}\right)=\frac{y\left(x_{i}\right) S\left(x_{i+1}\right)-y\left(x_{i+1}\right) S\left(x_{i}\right)}{y\left(x_{i+1}\right) C\left(x_{i}\right)-y\left(x_{i}\right) C\left(x_{i+1}\right)},
$$

where $S(x)=k x j_{l}(k x), \quad C(x)=k x n_{l}(k x)$ and $x_{i}<x_{i+1}$ both exist in the asymptotic region.

For positive energies and for $l=0$, we calculate the phase shift $\left(\delta_{l}\right)$ and then we compare it with the accurate value which is $\pi / 2$. The boundary conditions for this eigenvalue problem are $y(0)=0$ and $y(x)=\cos (\sqrt{E} x)$ for large $\mathrm{x}$.

We use the following eigenenergies

$E_{1}=53.588872$

$E_{2}=163.215341$

$E_{3}=341.495874$

$E_{4}=989.701916$

\subsection{BOUnd-STATES PROBlEM}

In the case of negative energies $(E<0)$, we consider the eigenvalue problem with boundary conditions $y(0)=0$ and $y(x)=\exp (-\sqrt{-E} x)$ for large $\mathrm{x}$.

In order to solve this problem numerically, by a chosen eigenvalue, we integrate forward from the point $x=0$, backward from the point $x=15$ and matching up the solution at some internal point in the range of integration.

For the Bound-states problem we use the following eigenenergies

$E_{1}=-49.457788728$

$E_{2}=-48.148430420$

$E_{3}=-41.232607772$

$E_{4}=-38.122785096$

$E_{5}=-26.873448915$

$E_{6}=-22.588602257$

$E_{7}=-8.676081670$

$E_{8}=-3.908232481$

We are going to compare our results to those derived by using the high order method of embedded Runge-Kutta-Nyström 4(3)4 method, of DEP (see [1]), as well as to other methods derived for the numerical solution of the Schrödinger equation. The methods used in the comparison have been denoted by:

- PAFRKN4: The new fourth-order RKN method with four stages (three effective stases with FSAL property), phase lag and amplification 


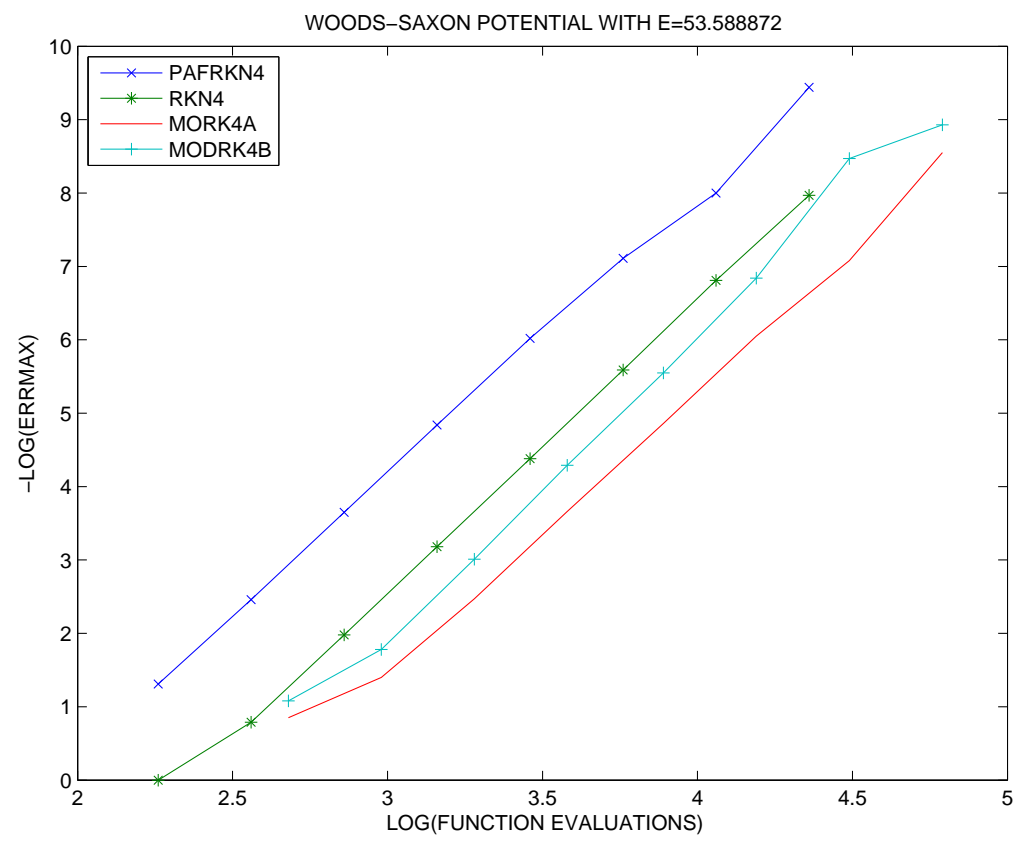

Figure 1. Efficiency for the Schrödinger equation using $\mathrm{E}=53.588872$

error of order infinity derived in Section 4.

- RKN4: The high order method of pair RKN 4(3)4 method of Dormand, El-Mikkawy and Prince [1].

- MODRK4A: The modified exponentially fitted fourth order RK method with four stages of $H$. Van de Vyver [5] (Section 2.1).

- MODRK4B: The modified exponentially fitted fourth order RK method with four stages of $H$. Van de Vyver [5] (Section 2.2).

One way to measure the efficiency of the method is to compute the accuracy in the decimal digits, that is $-\log _{10}$ (error at the end point) when comparing the phase shift to the actual value $\pi / 2$ versus the computational effort measured by the $\log _{10}$ (number of function evaluations required).

The frequency is given by the suggestion of Ixaru and Rizea [4]

$v= \begin{cases}\sqrt{E+50}, & x \in[0,6.5] \\ \sqrt{E}, & x \in[6.5,15]\end{cases}$

In the figures we display the efficiency curves, that is the accuracy versus the computational cost measured by the number of function evaluations required by each method. 


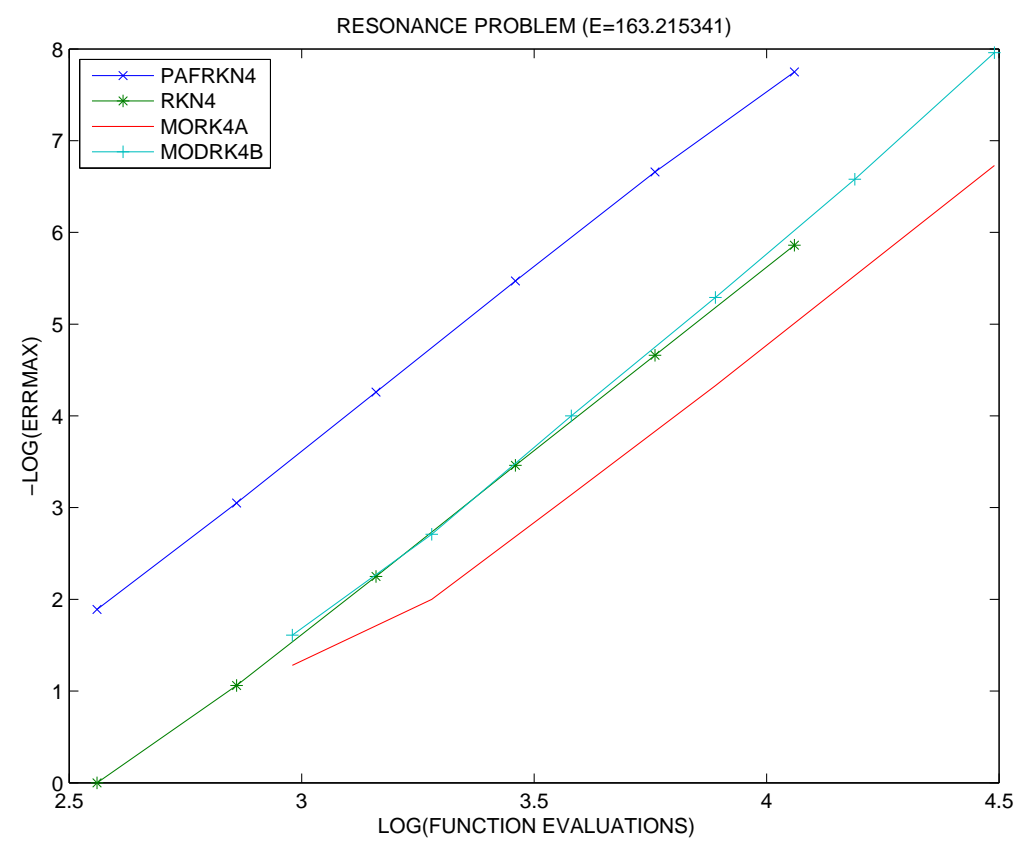

Figure 2. Efficiency for the Schrödinger equation using $\mathrm{E}=163.215341$

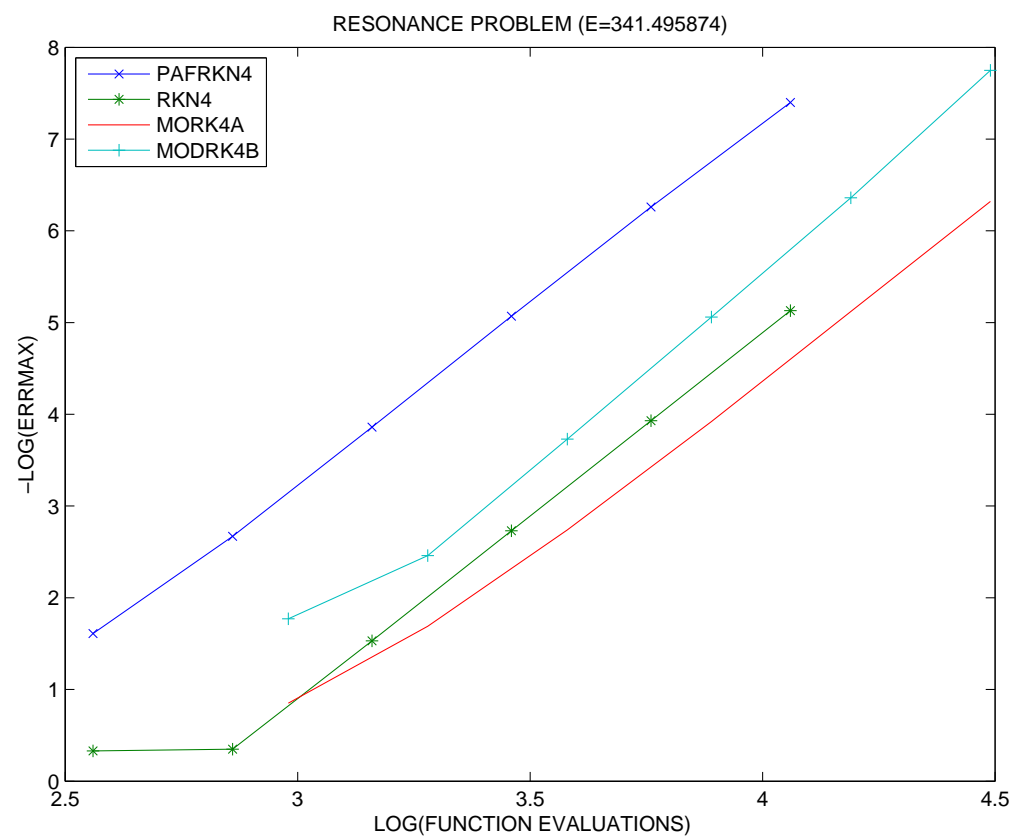

Figure 3. Efficiency for the Schrödinger equation using $\mathrm{E}=341.495874$ 


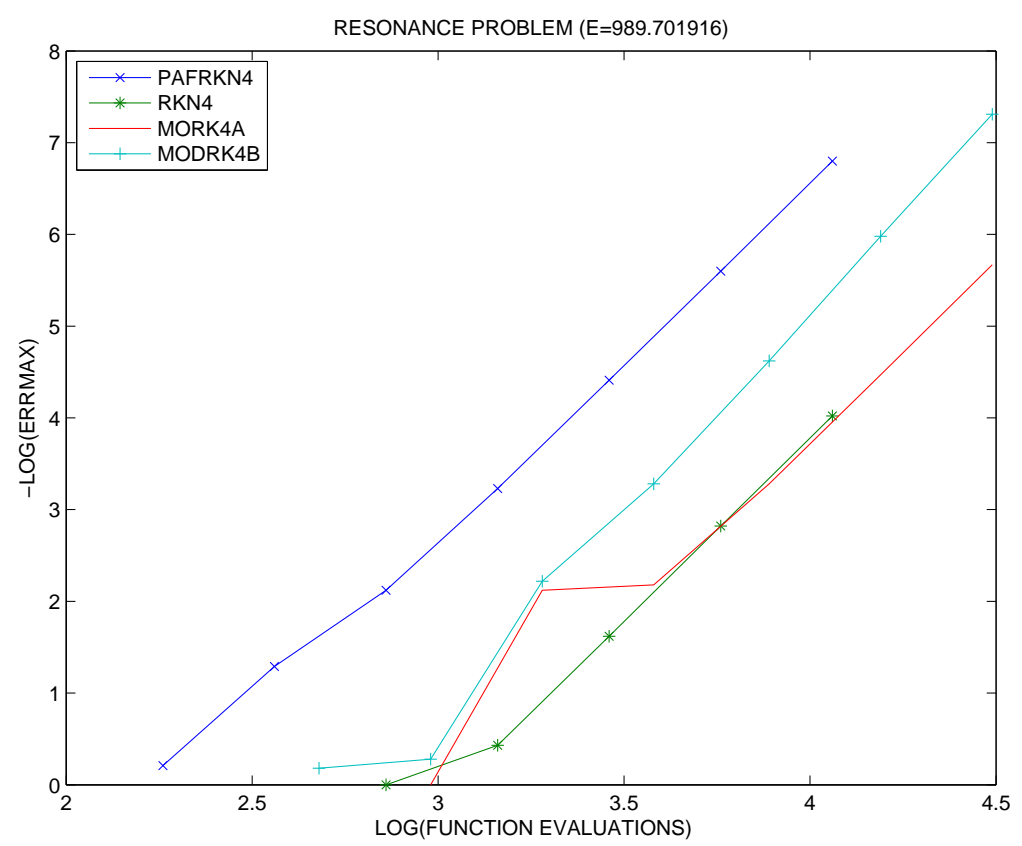

Figure 4. Efficiency for the Schrödinger equation using E=989.701916

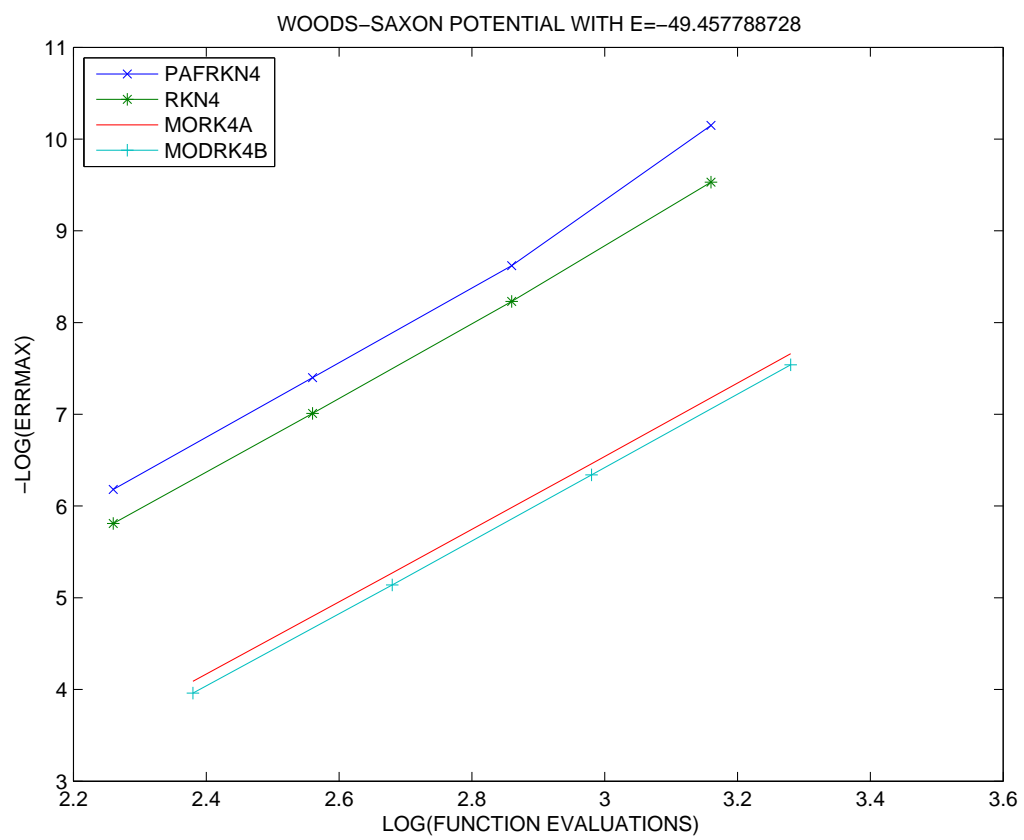

Figure 5. Efficiency for the Schrödinger equation using $\mathrm{E}=-49.457788728$ 


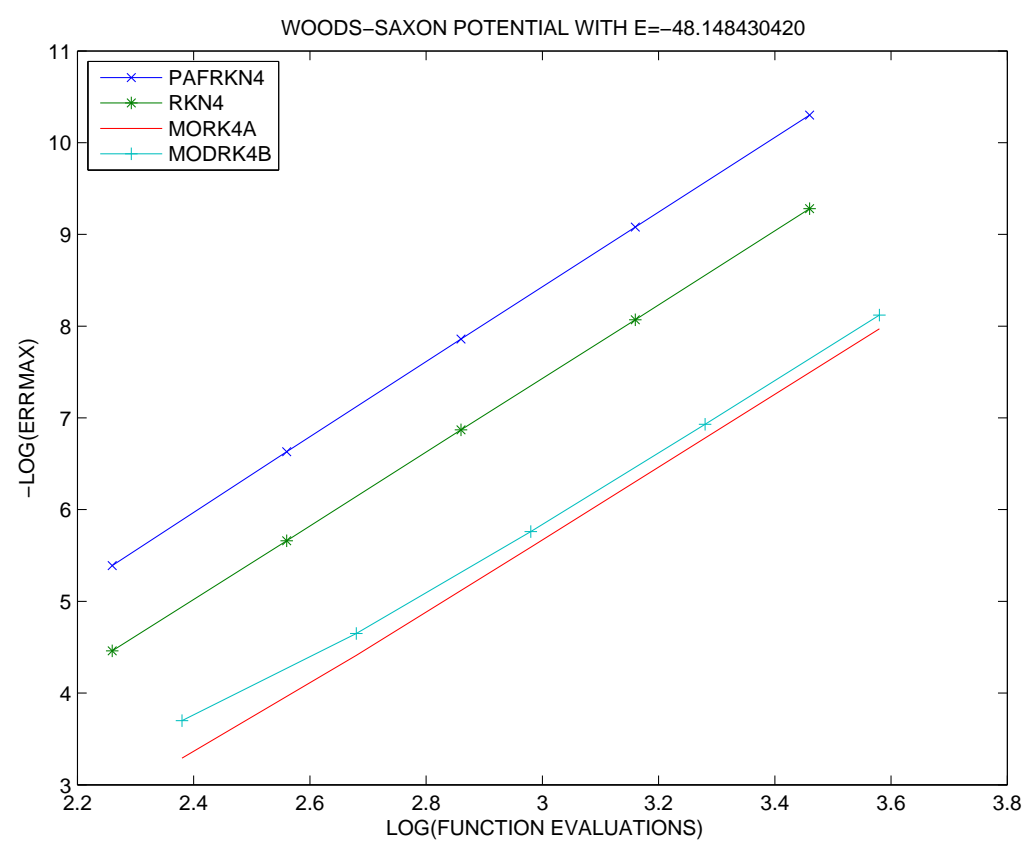

Figure 6. Efficiency for the Schrödinger equation using $\mathrm{E}=-48.148430420$

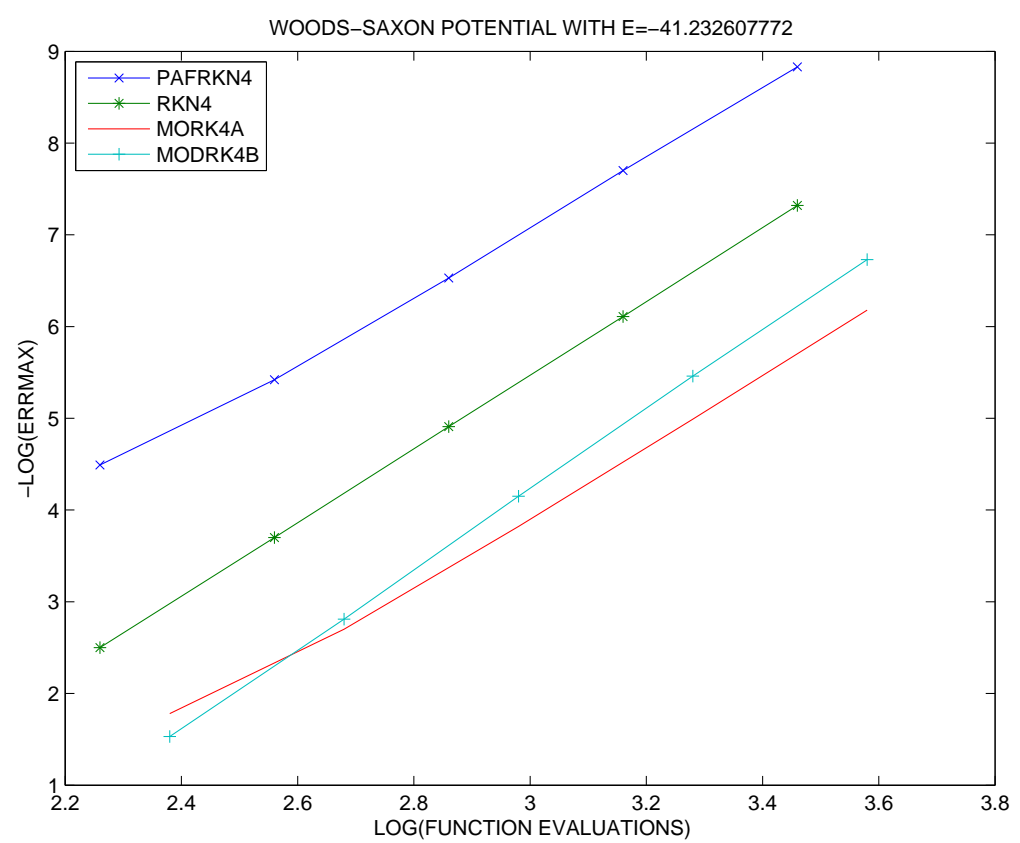

Figure 7. Efficiency for the Schrödinger equation using $\mathrm{E}=-41.232607772$ 


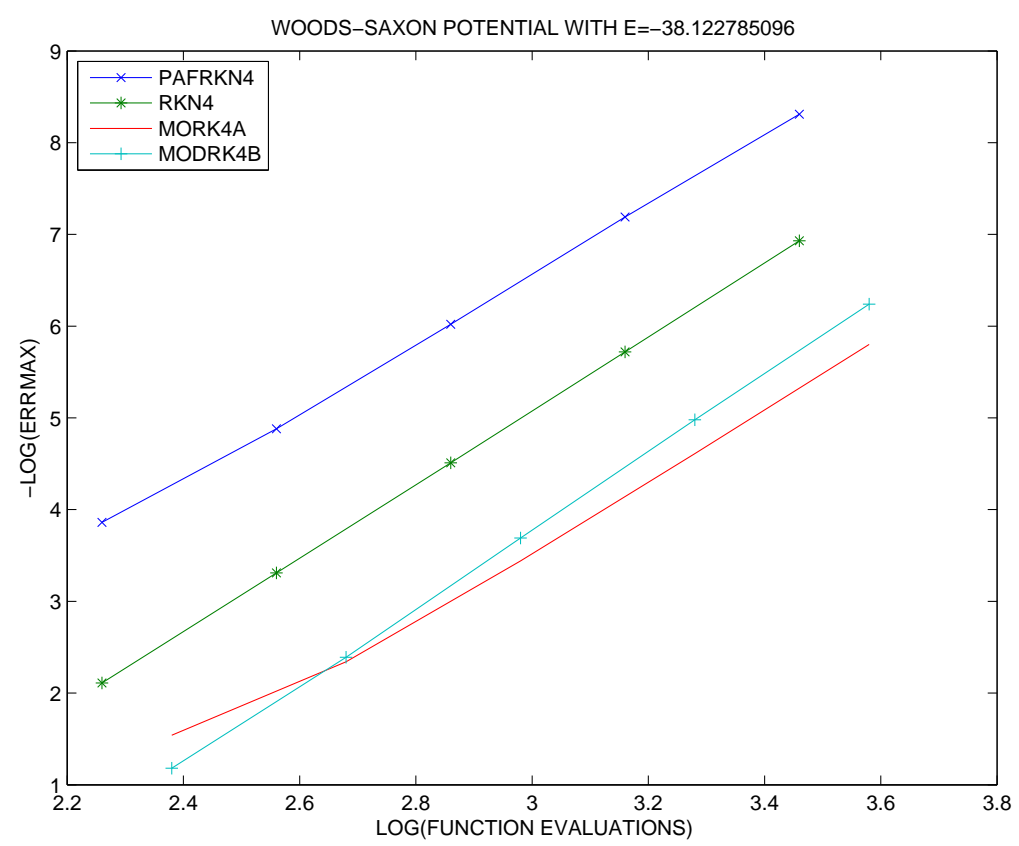

Figure 8. Efficiency for the Schrödinger equation using E=-38.122785096

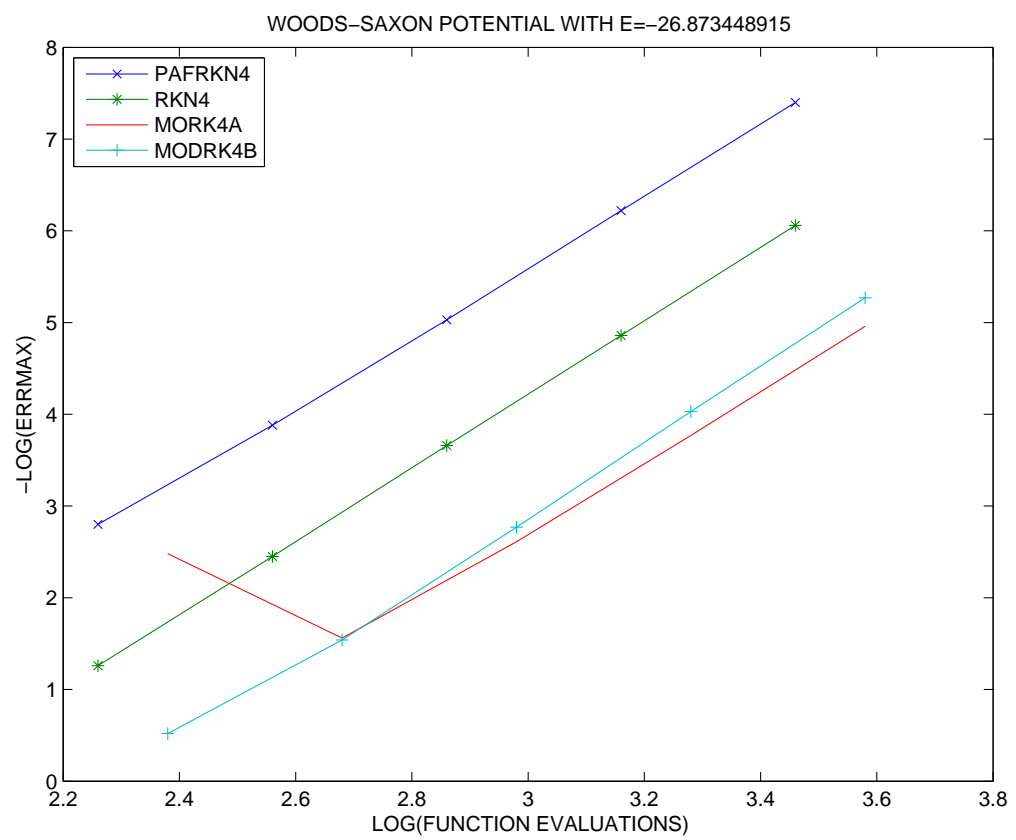

Figure 9. Efficiency for the Schrödinger equation using $\mathrm{E}=-26.873448915$ 


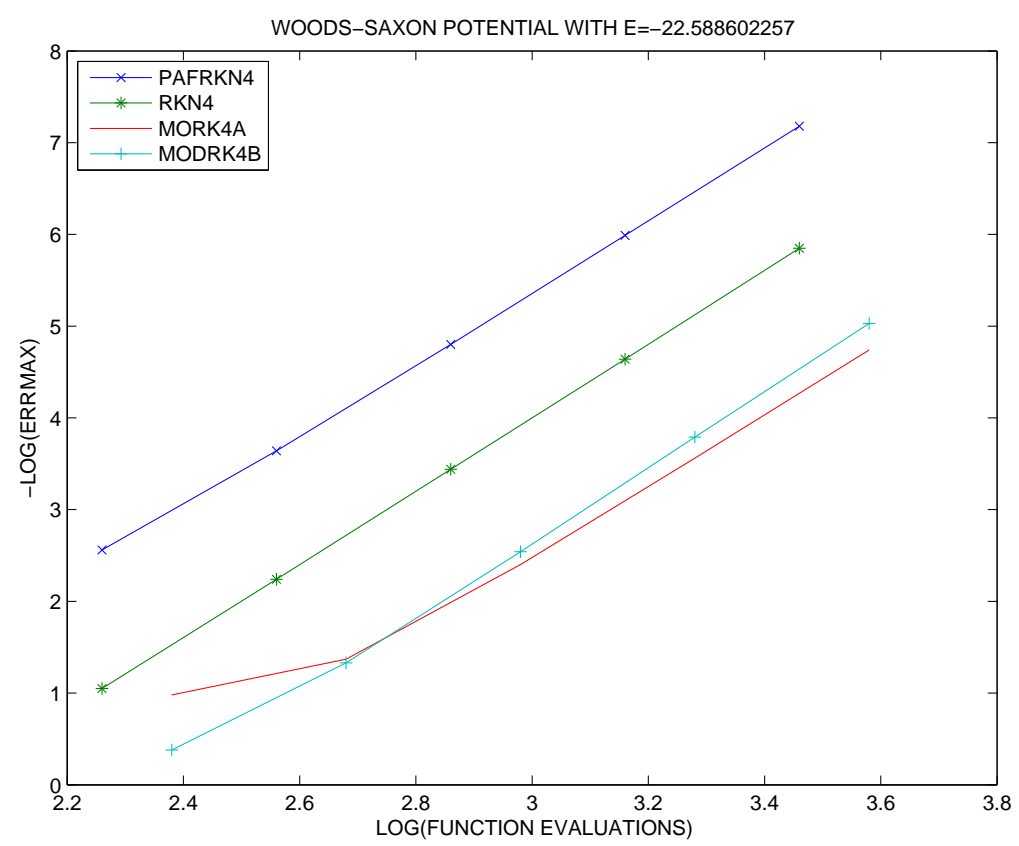

Figure 10. Efficiency for the Schrödinger equation using $\mathrm{E}=-22.588602257$

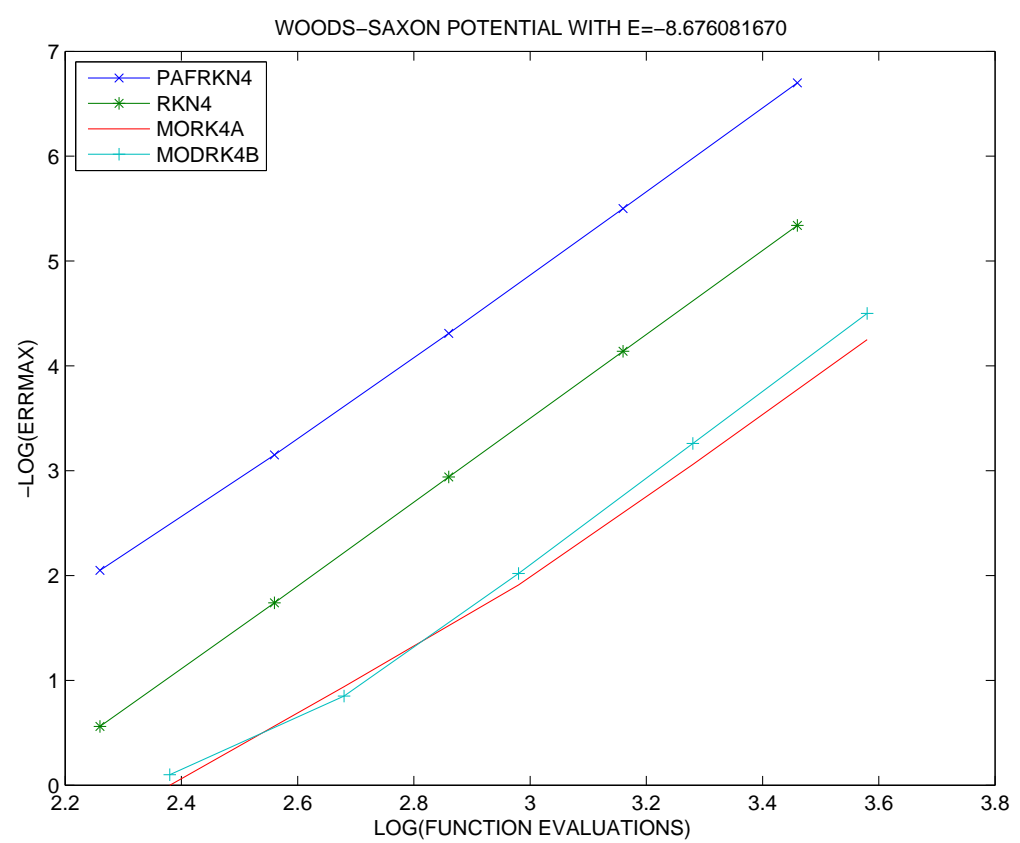

Figure 11. Efficiency for the Schrödinger equation using $\mathrm{E}=-8.676081670$ 


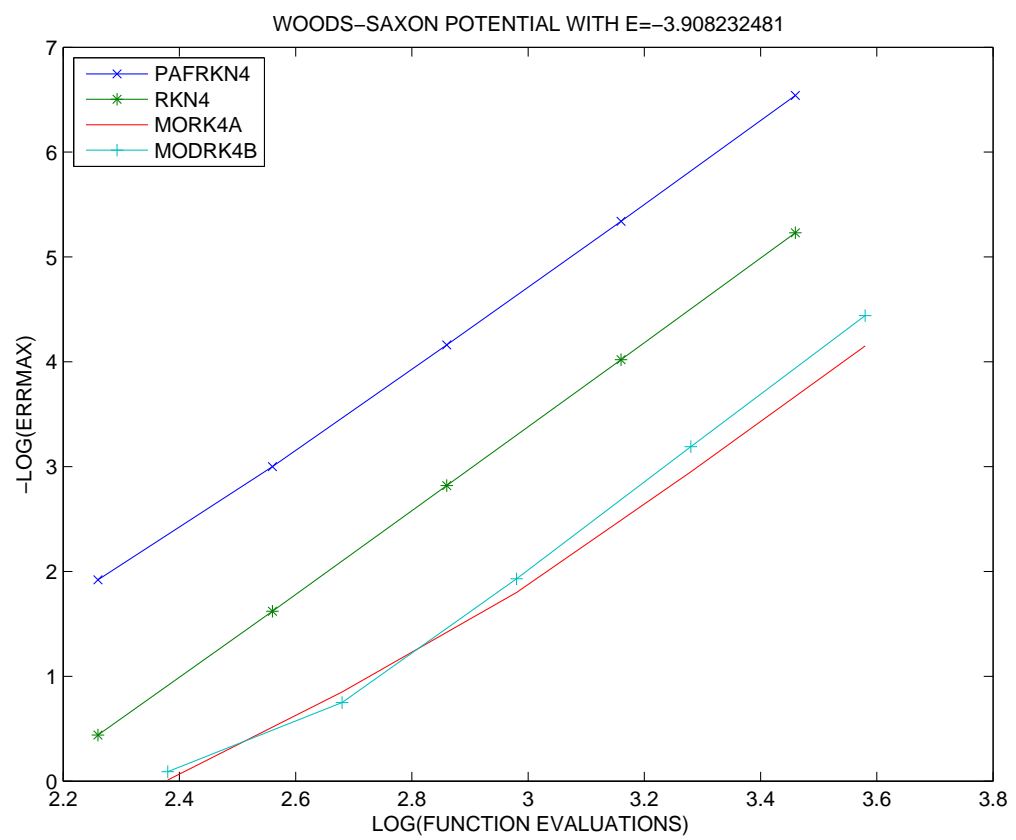

Figure 12. Efficiency for the Schrödinger equation using $\mathrm{E}=-3.908232481$

Numerical results indicate that the new method derived in section 4 , is very efficient for solving numerically the Schrödinger equation. More specifically we observe that, in the case of resonance problem our method is more accurate than MODRKB4 and MODRKA4 by two and three decimals respectively. Moreover the new method is more accurate by two decimals than RKN4 for the eigenvalues $E=$ $\{53.588872,163.215341,341.495874\}$ and by three decimals for the eigenvalue $E=989.701916$. In the case of bound-states problem, PAFRKN4 is more accurate than MODRKB4 and MODRKA4 by three decimals. Also our method maintained more accurate than the classical RKN4 method, by two decimals for the eigenvalues $E=\{-49.457788728$, $-48.148430420\}$ and by three decimals for the rest six eigenvalues.

\section{Conclusion}

A new Runge-Kutta-Nyström method of fourth algebraic order with four stages (three effective stages with FSAL property), is developed in the present paper. The new method is based on the very well known classical Dormand, El-Mikkawy and Prince fourth algebraic order RungeKutta-Nystöm method and it has phase lag of order infinity and ampli- 
fication error of order infintiy. The numerical results show that the new method is more efficient for the numerical solution of the Schrödinger equation than other methods derived for the same purpose, including the corresponding classical method of DEP.

\section{Acknowledgements}

We want to thank the anonymous reviewers for their careful reading of the manuscript and their fruitful comments and suggestions.

\section{References}

1. J.R. Dormand, M.E.A. El-Mikkawy and P.J. Prince, Families of Runge-KuttaNyström formulae, IMA J. Numer. Anal. 7 (1987) 235-250.

2. P.J. van der Houwen, B.P. Sommeijer, Explicit Runge-Kutta-Nyström methods with reduced phase errors for computing oscillating solutions, SIAM J. Numer. Anal. 24 (1987) 595-617.

3. John M. Blatt, Practical points concerning the solution of the Schrödinger equation, J. Comput. Phys. 1 (1967) 382-396.

4. L. Gr. Ixaru, M. Rizea, A numerov-like scheme for the numerical solution of the Schrödinger equation in the deep continuum spectrum of energies, Comput. Phys. Commun. 19 (1980) 23-27.

5. Hans Van de Vyver, Modified explicit Runge-Kutta methods for the numerical solution of the Schrödinger equation, Appl. Math. Comput. 171 (2005) 10251036.

6. Hans Van de Vyver, Comparison of some special optimized fourth-order RungeKutta methods for the numerical solution of the Schrödinger equation, Comput. Phys. Commun. 166 (2005) 109-122.

7. T. E. Simos, Jesu's Vigo Aguiar, A modified Runge-Kutta method with phaselag of order infinity for the numerical solution of the Schrödinger equation and related problems, Comput. Chem. 25 (2001) 275-281.

8. T.E. Simos and Jesus Vigo Aguiar, A Modified Phase-Fitted Runge-Kutta Method for the Numerical Solution of the Schrödinger Equation, J. Math Chem. 30 (2001) 121-131.

9. T.E. Simos, A family of fifth algebraic order trigonometrically fitted RungeKutta methods for the numerical solution of the Schrödinger equation, Comput. Materials Science 34 (2005) 342-354.

10. T. E. Simos, Exponentially fitted Runge-Kutta methods for the numerical solution of the Schrödinger equation and related problems, Comput. Materials Science 18 (2000) 315-332.

11. Z.A. Anastassi and T.E. Simos, Trigonometrically fitted Runge-Kutta methods for the numerical solution of the Schrödinger equation, J. Math. Chem. 37 (2005) 281-293. 
12. Z. A. Anastassi, D. S. Vlachos and T. E. Simos, A family of Runge-Kutta methods with zero phase-lag and derivatives for the numerical solution of the Schrödinger equation and related problems, J. Math. Chem. (2008).

13. Z. Kalogiratou, Th. Monovasilis, T.E. Simos, Computation of the eigenvalues of the Schrödinger equation by exponentially-fitted Runge-Kutta-Nyström methods, Comput. Phys. Commun. 180 (2009) 167-176.

14. Z. Kalogiratou and T.E. Simos, Construction of Trigonometrically and Exponentially Fitted Runge-Kutta-Nyström Methods for the Numerical Solution of the Schrödinger Equation and Related Problems - a Method of 8th Algebraic Order, J. Math. Chem. 31 (2002) 211-232. 
papdopoulos-anastassi-simos_rev.tex; 13/11/2009; 8:53; p.18 